\title{
Real-time observation of hidden multi-soliton dynamics in a few-cycle Ti:Sapph oscillator (Conference Presentation) (Withdrawal Notice)
}

Georg Herink, Felix Kurtz, Daniel Solli, Bahram Jalali, Claus Ropers

Georg Herink, Felix Kurtz, Daniel R. Solli, Bahram Jalali, Claus Ropers, "Real-time observation of hidden multi-soliton dynamics in a few-cycle Ti: Sapph oscillator (Conference Presentation) (Withdrawal Notice)," Proc. SPIE 10089, Real-time Measurements, Rogue Phenomena, and Single-Shot Applications II, 1008904 (12 February 2018); doi: 10.1117/12.2255659 


\title{
Real-time observation of hidden multi-soliton dynamics in a few-cycle Ti:Sapph oscillator (Conference Presentation) (Withdrawal Notice)
}

\author{
Proc. SPIE 10089, 1008904 (2017)
}

Online Publication Date: 21 April 2017

Withdrawn from Publication: 12 February 2018

Conference Date: 28 January-2 February 2017

Conference Location: San Francisco, California, United States

Conference Title: Real-time Measurements, Rogue Phenomena, and Single-Shot Applications II

Conference Chairs: Bahram Jalali, Daniel R. Solli, Sergei K. Turitsyn, Günter Steinmeyer, Neil G. R. Broderick

Georg Herink, Felix Kurtz, Georg-August-Univ. Göttingen (Germany); Daniel R. Solli, Bahram Jalali,

Claus Ropers, Univ. of California, Los Angeles (United States)

Publisher's Note: This conference presentation, originally published on 21 April 2017, was withdrawn per author request. 